ARTICLE

\title{
Large parental differences in chromatin organization in pancreatic beta cell line explaining diabetes susceptibility effects
}

Xing $\operatorname{Jian}^{1} \&$ Gary Felsenfeld (iD ${ }^{1 凶}$

Previous GWAS studies identified non-coding loci with parent-of-origin-specific effects on Type 2 diabetes susceptibility. Here we report the molecular basis for one such locus near the KRTAP5-6 gene on chromosome 11. We determine the pattern of long-range contacts between an enhancer in this locus and the human INS promoter $460 \mathrm{~kb}$ away, in the human pancreatic $\beta$-cell line, EndoC- $\beta \mathrm{H} 1$. $3 \mathrm{C}$ long range contact experiments distinguish contacts on the two sister chromosomes. Coupling with allele-specific SNPs allows construction of maps revealing marked differences in organization of the two sister chromosomes in the entire region between KRTAP5-6 and INS. Further mapping distinguishes maternal and paternal alleles. This reveals a domain of parent-of-origin-specific chromatin structure extending in the telomeric direction from the INS locus. This suggests more generally that imprinted loci may extend their influence over gene expression beyond those loci through long range chromatin structure, resulting in parent-of-origin-biased expression patterns over great distances.

\footnotetext{
${ }^{1}$ Laboratory of Molecular Biology, National Institute of Diabetes and Digestive and Kidney Diseases, National Institutes of Health, Bethesda, MD, USA.

凶email: gary.felsenfeld@nih.gov
} 
T he insulin (INS) locus on human chromosome 11 is embedded in a genomic region containing extended segments that are imprinted so that many of the surrounding genes are expressed in an allele-specific manner ${ }^{1,2}$. For example, in the IGF2/H19 locus, H19 imprinted maternally expressed transcript (H19) non-coding RNA is expressed from the maternal allele, whereas the insulin-like growth factor 2 (IGF2) gene is paternally expressed ${ }^{3,4}$. Toward the centromere end of this region, the potassium voltage-gated channel subfamily $\mathrm{Q}$ member 1 (KCNQ1) gene and the antisense KCNQ1-OT1 long non-coding RNA are expressed from only one allele ${ }^{5}$ (Fig. 1a).

Human INS expression itself is not imprinted, except in the yolk sac ${ }^{6}$. However, sites at the telomere end of chromosome 11 nearly $500 \mathrm{~kb}$ away near the gene keratin-associated protein 5-6 (KRTAP5-6) (Fig. 1b and Supplementary Fig. 1a) have been shown to harbor type 2 diabetes (T2D) susceptibility loci that display parent-of-origin-specific effects ${ }^{7}$. This raises the possibility that an extended chromosomal domain might have direct or indirect effects on INS expression, and INS expression might in turn affect expression of distant genes ${ }^{1,8,9}$.

To explore this possibility, it is necessary to identify, in INSexpressing cells, long-range and potentially chromosome of parent-of-origin-specific physical interactions between elements in the regions surrounding the INS gene. We used the human pancreatic $\beta$-cell line, EndoC- $\beta \mathrm{H}^{10,11}$, to focus initially on a region of chromosome $11 \sim 460 \mathrm{~kb}$ from INS, which has been reported ${ }^{7}$ to harbor a single nucleotide polymorphism (SNP), rs2334499, the T allele of which is associated with allele-specific effects on T2D susceptibility (Fig. 1a). This allele is linked with increased T2D incidence when paternally inherited but is protective when inherited maternally, indicating that some SNPs within the region confers an allele-specific phenotype affecting T2D susceptibility. In these cells rs2334499 is homozygous for the C (non-risk) allele (Supplementary Fig. 1b). SNP rs2334499 is located near the KRTAP5-6 gene and it was suggested ${ }^{7}$ that rs2334499 might be associated with transcriptional factor CTCF (CCCTC-binding factor) binding in a nearby regulatory element, OREG0020670 (Fig. 1a), and which in turn might be responsible for allele-specific behavior. In previous publications ${ }^{12-14}$, this locus was referred as $M O B$ Kinase Activator 2 (MOB2) or HCCA2. We find this nomenclature rather misleading, as in the current National Center for Biotechnology Information (NCBI) Homo sapiens Gene Annotation release 109.20210226 (https://www.ncbi.nlm.nih.gov/ genome/annotation_euk/Homo_sapiens/109.20210226/) the coding region of $M O B 2$ gene has been reduced from previously $295 \mathrm{~kb}$ to $17 \mathrm{~kb}$, so that rs2334499 and OREG0020670 is no longer within MOB2 gene. The element OREG0020670 has been characterized in a previous genomic study in EndoC- $\beta \mathrm{H} 1$ cells, as shown in Supplementary Fig. $2^{15}$ (also available at https://shinyapps.jax.org/ endoc-islet-multi-omics/). This element has the chromatin signature of an enhancer, and it is at the boundary between a euchromatic and a heterochromatic region.

Our earlier Chromosome Conformation Capture (3C) and Circularized Chromosome Conformation Capture (4C) measurements ${ }^{1}$ revealed strong contacts between the INS promoter and the KRTAP5-6 locus in EndoC- $\beta \mathrm{H} 1$ cell line. For this study, we also re-analyzed our $4 \mathrm{C}$ data ${ }^{1}$ with a newer analysis method, $4 \mathrm{C}-\mathrm{ker}^{16}$. As shown in Supplementary Fig. 3a and b, this is one of the strongest long-range interactions detected by our $4 \mathrm{C}$ data using the INS promoter as the bait sequence. When we decreased the $\mathrm{k}$-value (the number of observed fragments to be analyzed within each window) from 10 to 3 , we observed an increase of the strength of this contact. Meanwhile, the strength of most nearby contacts were decreased (Supplementary Fig. 3c). This new analysis is consistent with our previous analysis ${ }^{1}$. It is not known whether those long-range interactions occur on both chromosomes or preferentially on one, establishing a very long domain of allele-biased expression involving the INS gene indirectly or directly. Here we address this question by extended sequencing of the products of $3 \mathrm{C}$ experiments in EndoC- $\beta \mathrm{H} 1$ cells, coupled with identification of multiple allele-specific SNPs present in these cells. Combining these results allows us to identify a series of long-range contacts between the H19/IGF2 imprinted control region (H19-ICR) and the KRTAP5-6 locus, and sites between, that are confined in these cells to the maternal chromosome. We also map a contact between the INS promoter and KRTAP5-6 that is largely specific to the paternal chromosome. These two sites appear to mark the 5' and 3' boundaries of a single topologically associated domain (TAD). We identify a CTCF site within OREG0020670 that is differentially methylated

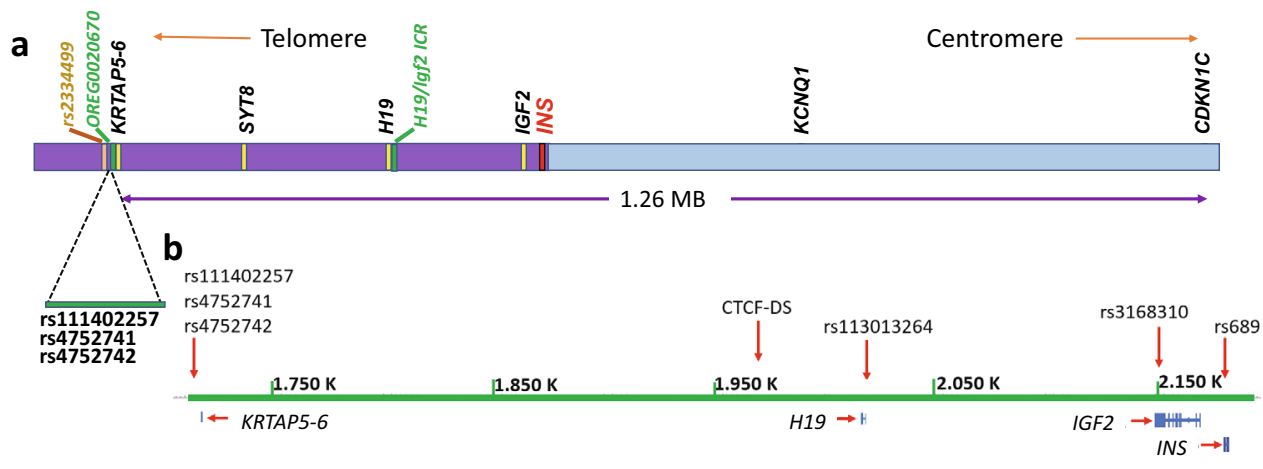

C Active INS in $\beta$ - cells

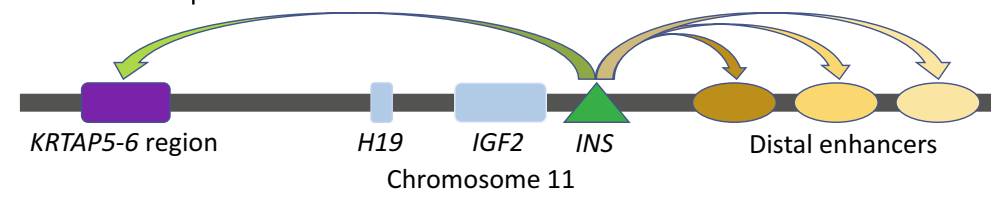

Fig. 1 Features of human chromosome 11p15.5-p15.4 region. a Schematic representation of the key genetic elements in this study, ranging from 1.6 Mb to 3.0 Mb (hg19 assembly) from the telomere-end of chromosome 11. The purple and blue bars represent approximate extents of distinct TADs in this region (see Discussion). b The genetic features mentioned in this study, on human chromosome 11, from 1.7 Mb to $2.2 \mathrm{Mb}$ (hg19 assembly). c The main $4 \mathrm{C}$-seq contact pattern of the INS promoter in EndoC- $\beta \mathrm{H} 1$ cells. There is one major contact to the KRTAP5- 6 region telomeric from the INS gene, whereas there are multiple major contacts to distal putative enhancers centromeric to the INS gene. 
and occupied in a chromosome specific fashion, consistent with a parent-of-origin-specific contact pattern. Finally, we use a SNP in an INS-IGF2 read-through transcript to show that in these cells INS-IGF2 is predominately expressed from the paternal allele. Our results establish a network of contacts extending over $460 \mathrm{~kb}$ that physically couples the INS locus to distant regulatory elements in a parent-of-origin-specific fashion. In principle, this can result in unequal expression of INS from the two alleles.

\section{Results}

SNP rs2334499 and its properties. Genome-wide association studies (GWAS) have identified the SNP rs2334499 (T/C) as a T2D susceptibility locus in humans ${ }^{7}$. Allele $\mathrm{T}$ of $\mathrm{rs} 2334499$, at 11p15.5 (Supplementary Table 1), shows only a weak association [Odds Ratio $(\mathrm{OR})=1.08, P=0.034]$ in the standard case-control test. However, when parental origin is considered, the paternally inherited allele $\left(\mathrm{OR}=1.35, P=4.7 \times 10^{-10}\right)$ makes a significant contribution to increased susceptibility. In contrast, the maternally inherited allele also shows significant association, but the effect of allele $\mathrm{T}$ is protective ( $\mathrm{OR}=0.86, P=0.002)$. Kong et al. identified a regulatory element OREG0020670, about $17 \mathrm{~kb}$ centromeric to rs 2334499 and $3 \mathrm{~kb}$ telomeric to the KRTAP5-6 gene, as a $2 \mathrm{~kb}$ region with multiple variably methylated $\mathrm{CpG}$ residues. The authors showed that the $\mathrm{T}$ allele was correlated with decreased methylation of these sites, regardless of parental origin. They suggested that CTCF binding to sites also present in this region might be controlled by $\mathrm{CpG}$ methylation, thus coupling long-range chromatin organization by CTCF to rs2334499.

We began by exploring the corresponding region in the human pancreatic $\beta$-cell line, EndoC- $\beta$ H1. Since OREG0020670 has been described as an enhancer in EndoC- $\beta \mathrm{H} 1$ cells (Supplementary Fig. 2) ${ }^{15}$, we decided to check whether deletion of the CTCF sites in this element would affect INS expression. We designed sgRNAs to target 3 CTCF motifs in this region (2 sgRNAs for each motif) by a recently developed CRISPR/Cas9 genome editing pipeline in EndoC- $\beta \mathrm{H} 1$ cells ${ }^{17}$. We quantified the editing events by a commercial T7-endonuclease I cleavage assay and found that editing most frequently occurred at the 5 '- and middle-CTCF sites $(24 \%$ and $27 \%)$, and the editing at the 3 '-CTCF site was not efficient (6\%) (Supplementary Fig. 4a, 5'-3' orientation as Fig. 1b). As recommended by this protocol, we co-transduced multiple sgRNAs simultaneously into EndoC- $\beta \mathrm{H} 1$ cells, and we also detected large deletions between the 5'- and middle-CTCF sites by performing a PCR with primers on either side of the sgRNA target sites ${ }^{17}$ (Supplementary Fig. 4b). We compared INS mRNA levels from the cells after the CTCF-site-targeting sgRNA editing and cells from non-target sgRNA control and found that INS expression was significantly reduced, by $28 \%$ (Supplementary Fig. $5, n=5$, Student's $t$ test, $t=3.9599, d f=8, p=0.0042$, mean $\Delta \Delta \mathrm{Ct}=0.47,95 \%$ confidence interval of $\Delta \Delta \mathrm{Ct}: 0.20-0.74,95 \%$ confidence interval of expression change: $0.60-0.87)$. As the current protocol cannot produce single clones of cells due to the difficult cell growth condition, we have not performed further functional studies on OREG0020670.

KRTAP5-6 and the INS locus. Although the KRTAP5-6 locus (and OREG0020670) is about $460 \mathrm{~kb}$ distant from the INS promoter, our earlier $4 \mathrm{C}$-seq results showed that in EndoC- $\beta \mathrm{H} 1$ cells there are strong contacts between these two sites on chromosome 11 (Fig. 1c and Supplementary Fig. 3) ${ }^{1}$. There are multiple other interactions between the INS promoter and sites in both directions along the chromosomes. Expression of many genes in this region is parent-of-origin-specific, and this raises the question whether the entire large domain from KRTAP5-6 to sites centromeric to INS might be connected by a system of such specific contacts that could help control expression. We therefore made a series of measurements designed to determine whether the distant contacts identified by $4 \mathrm{C}$-seq occurred equally on both alleles or biased toward one, and if the latter, whether in each case the contacts were preferentially on the maternal or paternal alleles.

We carried out 3C-PCR analyses to determine interactions among sites within the entire region between OREG0020670 and the INS promoter, taking advantage of the presence of three heterozygotic SNPs, [rs111402257(C/-C), rs4752741 (G/A), rs4752742(A/G); Fig. 1a/b, and Supplementary Fig. 6] to distinguish between contact patterns on the two alleles.

We first analyzed the products of $3 \mathrm{C}$ experiments using sites near INS and KRTAP5-6 as anchors. The three SNPs in the KRTAP5-6 region described above allowed us to distinguish alleles. Direct sequencing of the products revealed an $\sim 2.5: 1$ bias in favor of the $+\mathrm{C} / \mathrm{G} / \mathrm{A}$ over the $-\mathrm{C} / \mathrm{A} / \mathrm{G}$ allele (Fig. 2a). This was confirmed by sequencing individual clones of the 3C products; 19 out of 21 clones contained the $+\mathrm{C} / \mathrm{G} / \mathrm{A}$ allele (Supplementary Fig. 7). On the other hand, PCR from a 1:1 mixture of synthetic $3 \mathrm{C}$ templates only showed slight amplification bias ( 0.52:0.48) toward $+\mathrm{C} / \mathrm{G} / \mathrm{A}$ allele over $-\mathrm{C} / \mathrm{A} / \mathrm{G}$ allele (Supplementary Fig. 8). Thus, this long-range contact between INS and KRTAP5-6 occurs preferentially on one of the two alleles. This observed bias in contact frequency cannot be attributed to the rs2334499 polymorphism because it is homozygous in EndoC- $\beta \mathrm{H} 1$ cells.

The question raised by these results is whether the specific contacts in the KRTAP5-6 neighborhood are associated with the maternal or paternal allele. To determine this, we focused on the IGF2/H19 locus about $300 \mathrm{~kb}$ centromeric to KRTAP5-6. Much of the underlying pattern of $3 \mathrm{C}$ contacts of this region has been described $^{18}$ and the parent-of-origin-specific expression behavior is well documented ${ }^{5,19,20}$. H19 is expressed primarily from the maternal allele and contacts between the $H 19$ enhancer and other relatively nearby sites are allele specific ${ }^{18}$. To map the contacts in this region we identified the (A/G) SNP rs113013264 (Fig. 2b), about $2 \mathrm{~kb}$ centromeric to $H 19$ transcription start site, located within the H19-ICR. Sequencing of 3C data anchored in the H19 promoter and enhancer showed a strong bias for the $G$ allele, identifying this allele as maternal (Fig. 2b). To support this assignment, we made use of the observation ${ }^{18}$ that the interaction between H19-ICR and a distal CTCF-binding site (CTCF-DS, $\sim 50 \mathrm{~kb}$ away in the telomere direction) occurs mostly in the maternal allele in human B3 breast cancer cells. Examination of 3C contacts between these two sites (Fig. 2b) again shows that the $\mathrm{G}$ allele of rs113013264 preferentially contacts the CTCF-DS site (10/13G allele in cloned sequences, and $>95 \%$ G over A allele in the $3 \mathrm{C}$ product), indicating this as the maternal allele (Fig. $2 \mathrm{~b}$ and Supplementary Fig. 9). We also analyzed the methylation pattern of H19-ICR, as rs113013264 is located at one of the CTCFbinding site called hyper-sensitive site 5 (HS5). The A allele of rs113013264 would lack a CpG in HS5, which has been shown not affecting CTCF binding ${ }^{21}$, and is not conserved in other CTCF-binding sites at H19-ICR locus ${ }^{19}$. We obtained 17 clones of the G allele and detected fully unmethylated HS5 in all of them (Supplementary Fig. 10a). On the other hand, for the 4 clones of A allele obtained, we detected fully methylated HS5 (Supplementary Fig. 10b). This result indicates that H19-ICR has normal imprinted methylation pattern in EndoC- $\beta \mathrm{H} 1$ cells. It also confirmed again that $G$ allele of rs113013264 is the maternal allele, and A allele is the paternal allele.

With this information we were able to determine the contact pattern of the KRTAP5-6 locus. Making use again of rs113013264 we analyzed $3 \mathrm{C}$ products anchored in the neighborhoods of the H19-ICR and sites containing the three SNPs (rs111402257, rs4752741, rs4752742) in the KRTAP5-6 locus. All (9 out of 9) obtained 3C products contained both the G allele near the H19-ICR 


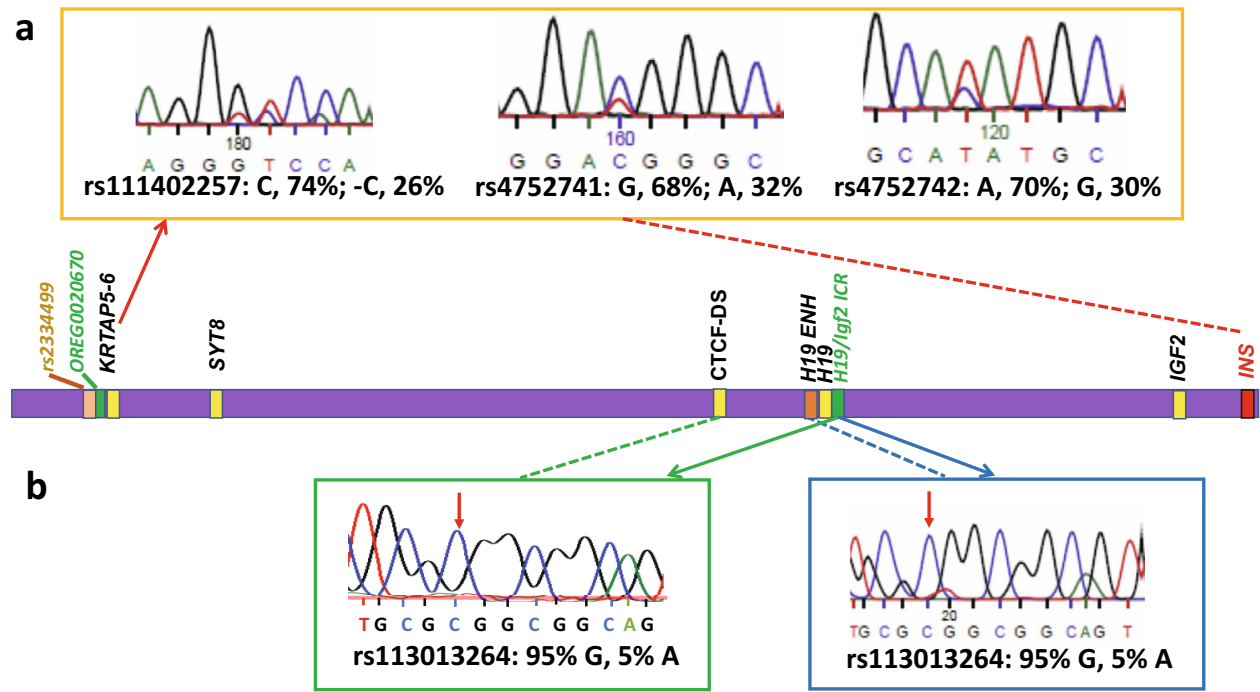

Fig. 2 3C SNP analysis showing allele-specific chromatin contact patterns. a The contact between INS and OREG0020670 near the KRTAP5-6 gene is enriched with allele rs 111402257 (C)/rs4752741(G)/rs4752742(A) over allele rs $111402257(-\mathrm{C}) / \mathrm{rs} 4752741(\mathrm{~A}) / \mathrm{rs} 4752742(\mathrm{G})$. The sequencing read is from the reverse strand. $\mathbf{b}$ The known mono-allelic contact pattern in the neighborhood of $\mathrm{H} 19$ is conserved in EndoC- $\beta \mathrm{H} 1$ cells. Left: the contact between H19-ICR and CTCF-DS is enriched with the G allele of rs113013264 over A allele. The sequencing read is from the reverse strand. Right: the contact between H19-ICR and H19 enhancer is enriched with G allele of rs113013264 over A allele. The sequencing read is from the reverse strand. Color coded arrows and dashed lines show contact patterns.

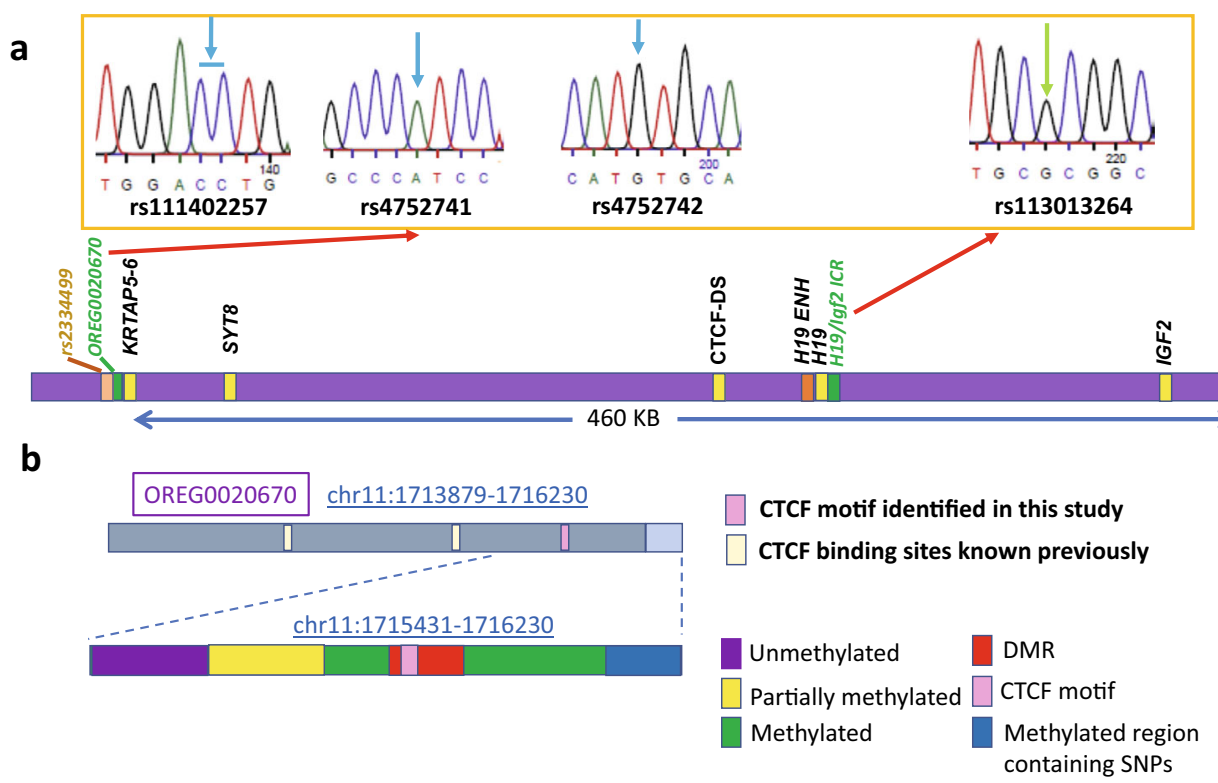

Fig. 3 Assignment of the parental origin of the allele-specific contacts. a Sequencing read of a single clone of 3C-PCR product between H19-ICR and OREG0020670 near the KRTAP5-6 gene showed that it contains G allele (marked with green arrow) of H19-ICR (rs113013264) and -C/A/G allele (marked with blue arrows) of OREG0020670 (rs111402257/rs4752741/rs4752742). The sequencing read is from the reverse strand for H19-ICR and from the forward strand for OREG0020670. Red arrows show contact sites. Sequencing results of eight additional clones of this 3C-PCR product is shown in Supplementary Fig. 11. b Key features of OREG0020670. Dark gray: euchromatin. Light grey: heterochromatin. Previously and newly identified CTCF sites $^{22}$ are marked by small boxes. Enlarged bar graph represents the CpG methylation status in this region. Pink: CTCF motif (see Supplementary Figs. 6 and 14). Blue: methylated region containing SNPs rs111402257, rs4752741 and rs4752742. Purple: unmethylated region. Yellow: Variable methylated region without strong allelic specificity. Red: Differentially methylated region with allelic specificity. Green: methylated region. The DNA sequence (hg19 chromosome 11: 1715431-1716230) is shown in detail in Supplementary Fig. 6.

and the $-\mathrm{C} / \mathrm{A} / \mathrm{G}$ allele in the KRTAP5-6 locus (Fig. 3a and Supplementary Fig. 11), establishing that the $-\mathrm{C} / \mathrm{A} / \mathrm{G}$ allele, and this contact, are maternal. To confirm this assignment, we also analyzed two 3C products containing SNP rs113013264 at the H19ICR but without SNPs at the KRTAP5-6 locus (Supplementary
Fig. 12). Both products also contained predominately the $G$ allele at the H19-ICR (10 out of 10 cloned sequences, $>99 \%$ G over A allele in the first $3 \mathrm{C}$ product, and $>95 \% \mathrm{G}$ over A allele in the second $3 \mathrm{C}$ product, Supplementary Figs. 12 and 13). We think this bias is due to the methylation of the paternal H19-ICR, which prevents CTCF- 


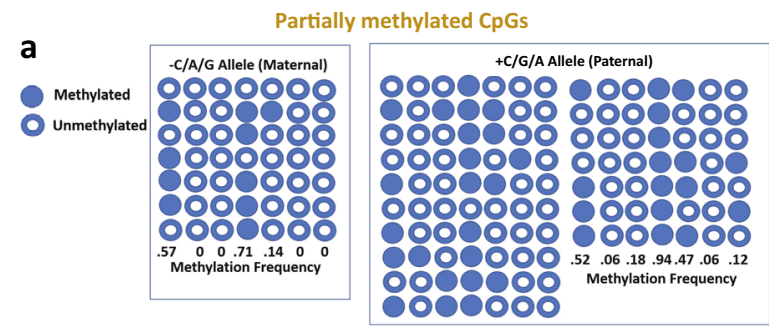

b

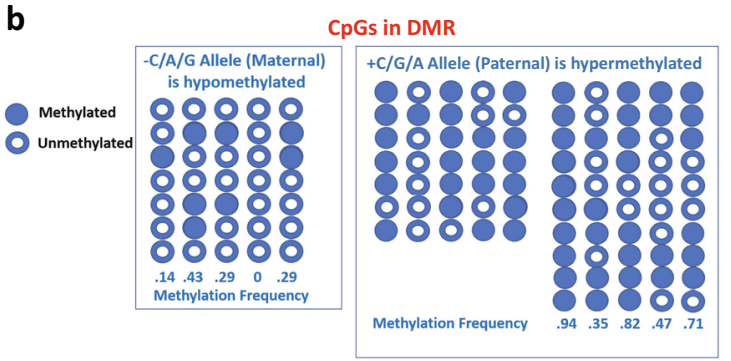

C
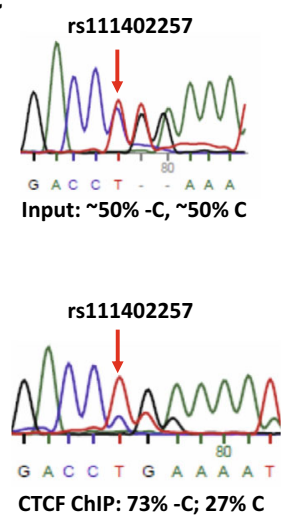

Fig. 4 OREG0020670 shows allele-specific methylation and CTCF occupancy. a The seven CpGs in the variable methylated region (orange-colored CpGs in Supplementary Fig. 6) are partially methylated without strong allelic preference. b The CpGs in differentially methylated region (red-colored CpGs in Supplementary Fig. 6) are partially methylated with strong preference in the paternal allele, except for the second CpG. c. ChIP-PCR shows that CTCF preferentially binds to the maternal allele ( $-\mathrm{C}$ allele of rs111402257). Top: genomic input sample. Bottom: CTCF-ChIP sample.

binding. This can be contrasted with the interaction determined above between the INS promoter and KRTAP5-6 regions (Fig. 2a), which these results show to be specific principally to the paternal allele (C/G/A allele) at KRTAP5-6 locus.

CpG methylation patterns at OREG0020670. We carried out sequencing and bisulfite analysis of the region chr11:1715431-1716230 (Fig. 3b), at the centromeric end of OREG0020670, taking advantage of the presence of three SNPs mentioned above, [rs111402257 (C/-C), rs4752741 (G/A), rs4752742 (A/G); Fig. 1] to distinguish between methylation patterns on the two alleles. These patterns (Fig. 4, Supplementary Fig. 6) vary: At the 5' end of the sequence the $\mathrm{CpG}$ residues are unmethylated, but, in the region immediately downstream, methylation is partial, and randomly distributed between alleles, except for a single site that is highly methylated on both alleles (Fig. 4a, orange-colored CpGs in Supplementary Fig. 6). Beyond this we find a region extending for at least $500 \mathrm{bp}$ within which levels of $\mathrm{CpG}$ methylation are high. Within this region, we find a CpG island showing an allele-specific methylation pattern, indicating a differentially methylated region (DMR): the $+\mathrm{C} / \mathrm{G} / \mathrm{A}$ allele is extensively methylated at a few $\mathrm{CpG}$ sites, whereas the $-\mathrm{C} / \mathrm{A} / \mathrm{G}$ is methylated at much lower levels (Fig. $4 \mathrm{~b}$, red-colored CpGs in Supplementary Fig. 6). we analyzed the sequence of this DMR with CTCFBSDB 2.0 (https://insulatordb.uthsc.edu/) ${ }^{22}$, and identified a CTCF-binding motif inside (Fig. 4c, Supplementary Figs. 6 and 14) ${ }^{23}$. Chromatin immunoprecipitation followed by PCR (ChIP-PCR) in EndoC- $\beta \mathrm{H} 1$ cells showed that CTCF preferentially binds to this site in vivo on the chromosome carrying the $-\mathrm{C} / \mathrm{A} / \mathrm{G}$ allele, which is the hypomethylated allele, and as shown above is the maternal allele. The binding motif for this CTCF site is immediately adjacent to, but does not overlap with, a methylatable CpG (Supplementary Fig. 6). We found that the CTCF zinc finger domain (containing all the eleven zinc fingers) could bind both the methylated and the unmethylated doublestrand DNA (dsDNA) sequence in vitro (Supplementary Fig. 15), which is not surprising, as the CpGs are not within the CTCFbinding motif (Supplementary Fig. 6). The observed allele specificity of binding may not depend directly upon the methylation status of this site, and it may be connected to the chromatin structure of the entire region (see Discussion).

Paternally biased expression of INS-IGF2 transcript. We hypothesized that the difference in chromosome contact pattern between the two parental alleles might affect the transcription activity of the INS promoter. The INS gene is homozygotic in EndoC- $\beta \mathrm{H} 1$ cells, so we could not determine whether there is any difference in the INS mRNA abundance between the two parental alleles. Since EndoC- $\beta \mathrm{H} 1$ cells only grow in high cell density, and form islet-like organoids, we were unable to introduce heterozygotic SNPs in the INS gene in an allele-specific manner by a conventional single-colony screening method. Instead, we decided to measure the INS promoter activity from an INS-IGF2 readthrough transcript. When the INS gene is transcribed in human cells, a small fraction of the mRNA fails to terminate at the 3 '-end of the gene. Instead, the transcript is extended into the downstream IGF2 gene (Fig. 5a). This transcript is expressed in a highly tissue-specific pattern: only in the pancreas is it expressed bi-allelically ${ }^{24}$. INS-IGF2 was reported to be up-regulated in insulinomas ${ }^{25}$. There are two mature isoforms produced from this INS-IGF2 read-through transcript, and it is still under debate whether the isoform 2 is involved in autoimmunity of type 1 diabetes (T1D) ${ }^{26-29}$.

The isoform 1 terminates at the 3 '-end of the IGF2 gene and is formed by splicing between the second exon of INS and the first exon of IGF2. We found that this isoform contains a heterozygotic SNP rs3168310 (G/C) at the last exon (Supplementary Fig. 16). We were able to amplify a fragment of this isoform by RT-PCR from the total RNA of EndoC- $\beta \mathrm{H} 1$ cells. The PCR primers are located within the 2nd exon of INS and the last exon of IGF2, so that only INS-IGF2 read-through transcript would be amplified. Sequencing this PCR product showed that at SNP rs3168310, the $\mathrm{G}$ allele is the major product (71.9\%) relative to the $\mathrm{C}$ allele (28.1\%) (Fig. 5b). Thus, the isoform 1 of INS-IGF2 is preferentially expressed from the $\mathrm{G}$ allele in EndoC- $\beta \mathrm{H} 1$ cells. In the next step, we determined the parental origin for the $G / C$ allele at SNP rs3168310. We successfully obtained 3C-PCR product that contains both rs113013264 in H19-ICR and rs3168310 in IGF2 (Fig. 5c-f). By sequencing individual clones 


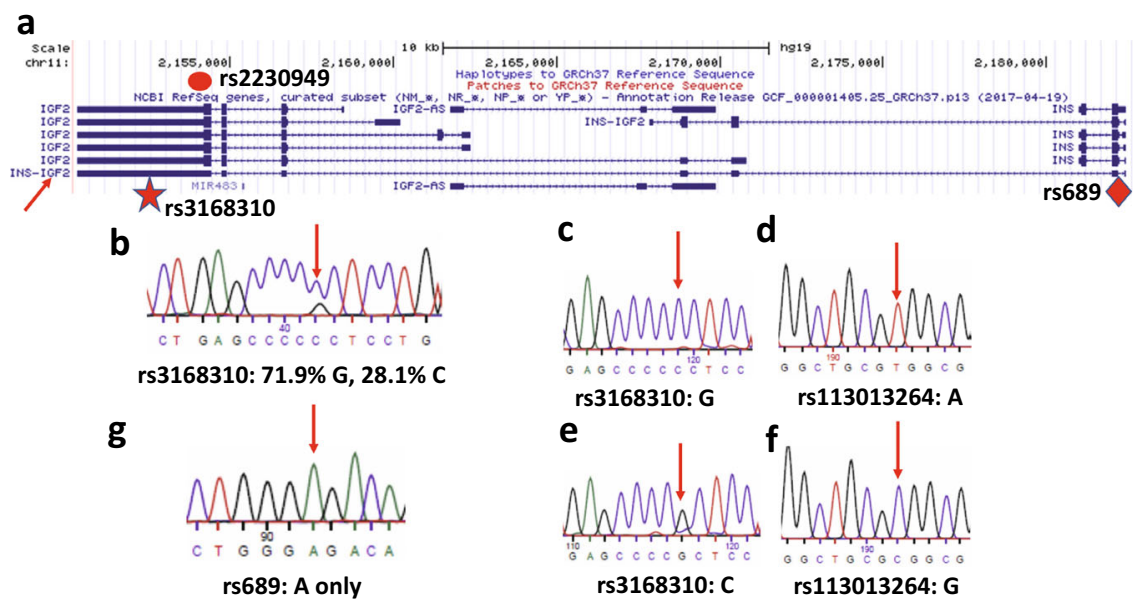

Fig. 5 Paternally biased expression of INS-IGF2 transcript. a Gene isoforms at IGF2-INS locus. Data is retrieved from UCSC Genome Browser ${ }^{42}$ hg19 assembly from chr11:2150251-2182750. Approximate position of SNP rs3168310 is marked with a star in the last exon of the IGF2 gene, and that of SNP rs689 with a diamond in the first intron of the INS gene. Isoform 1 of INS-IGF2 transcript is marked with an arrow. b Sequencing INS-IGF2 isoform 1 PCR product shows that at SNP rs 3168310 , the $\mathrm{G}$ allele is the major product over $\mathrm{C}$ allele $(71.9 \% \mathrm{G}, 28.1 \% \mathrm{C}$ ). Sequencing primer reads from the reverse strand. c-f SNP typing H19-ICR-IGF2 3 C product. The IGF2 rs3168310 G allele (c) is found in the same PCR product with the H19-ICR rs113013264 A allele (d). Meanwhile, the IGF2 rs3168310 C allele (e) is found in the same PCR product with the H19-ICR rs113013264 G allele (f). $\mathbf{g}$ Genotyping of rs689. Sanger sequencing showed that in EndoC- $\beta \mathrm{H} 1$ cells, SNP rs689 is the monoallelic A allele. The sequencing primer reads from the forward strand.

of this product, we found that the IGF2 rs $3168310 \mathrm{G}$ allele is in the same PCR product with the H19-ICR rs113013264 A allele (Fig. 5c, d), and the IGF2 rs3168310 C allele is in the same PCR product with the H19-ICR rs113013264 G allele (Fig. 5e, f). Since we already know that for rs113013264, A allele is paternal and G allele is maternal, we assign the $\mathrm{G}$ allele for rs3168310 to be paternal, and the $\mathrm{C}$ allele to be maternal. This assignment also agrees with the assumption that IGF2 is predominately expressed from the paternal allele, as we obtain 5 times more clones of the 3C-PCR product from the assigned paternal allele than from the maternal allele. This assignment indicates that the isoform 1 of INS-IGF2 is preferentially expressed from the paternal allele relative to the maternal allele. It is known that the variable number tandem repeat (VNTR) minisatellite polymorphism at the INS promoter would affect the expression of INS mRNA level ${ }^{30}$. This VNTR consensus sequence contains a binding site for transcription factor Myc-associated zinc finger protein $(\mathrm{MAZ})^{31,32}$, which was recently reported to interact with $\mathrm{CTCF}^{33}$. We genotyped the nearby SNP rs689 which is in tight linkage disequilibrium with the INS VNTR $(>99 \%$ in the Caucasian population $)^{34}$. The sequencing results showed that rs689 is the homozygotic A allele (Fig. 5g), which indicates that in both the INS promoters, the INS VNTR class I allele is present. Thus, the large allelic bias we found in the INS-IGF2 transcript level is not due to the INS VNTR polymorphism.

We obtained a human pancreatic islet sample to test whether paternally biased expression of INS-IGF2 would occur in mature $\beta$-cells. The genotyping from genomic DNA showed that rs2230949 is a heterozygotic G/A locus in this sample (Fig. 6a). The sequencing result of IGF2 isoform 1 transcript from RT-PCR showed that only the A allele of rs2230949 is expressed, indicating that this allele is the paternal allele (Fig. 6b). When we sequenced the INS-IGF2 isoform 1, we found that the A allele is preferentially expressed $(59.8 \pm 1.9 \%$, from 3 portions of the sample, Fig. 6c, Supplementary Fig. 17). The sequencing results showed that rs689 is the homozygotic $\mathrm{T}$ allele in this sample (Fig. 6d), indicating that the INS VNTR class III allele is present in both INS promoters. In this human sample, it seems INS-IGF2 is expressed preferentially from the paternal allele, matching with our observation in EndoC- $\beta \mathrm{H} 1$ cells. a

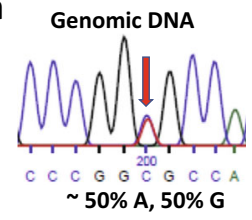

C

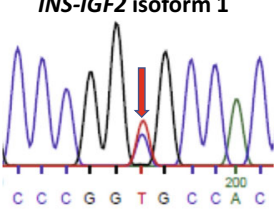

$59.9 \%$ A, $40.1 \%$ G b IGF2 isoform 1

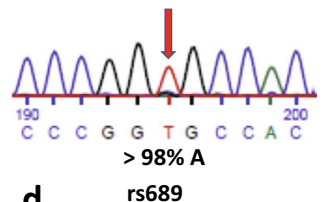

d

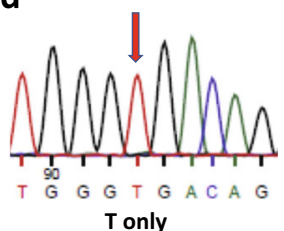

Fig. 6 Allelic biased expression of IGF2 and INS-IGF2 in a human pancreatic islet sample. The sequencing primer reads from the reverse strand in a-c, and from the forward strand in d. a Genotyping of rs2230949 from genomic DNA, locating inside the last exon of IGF2 gene. The locus is a G/A heterozygote. b Genotyping of rs2230949 of IGF2 isoform 1 transcript. Only the $A$ allele is expressed, indicating that this is the paternal allele. c Genotyping of rs 2230949 of INS-IGF2 isoform 1 transcript. The expression is biased toward the A allele. d Genotyping of rs689, showing that rs689 is the monoallelic T allele.

\section{Discussion}

By combining identification of allele-specific SNPs with extended sequencing of the products of $3 \mathrm{C}$ experiments, we have identified a large domain in a human pancreatic $\beta$-cell line containing the INS gene, in which chromatin organization is allele specific. This domain encompasses the KRTAP5-6 locus at one end and the INS locus at the other, a distance of $\sim 460 \mathrm{~kb}$. The KRTAP5- 6 gene encodes a keratin-associated protein as a component of hair matrix $^{35,36}$, which has not been reported to be related to diabetes. There is also no information about whether the SNP rs2334499 would affect its expression. This gene is expressed mainly in liver, placenta and skin, and has not been detected in pancreas ${ }^{37}$ (https://www.proteinatlas.org/ENSG00000205864-KRTAP5-6/ tissue). Consistent with the presence of a domain boundary telomeric to KRTAP5-6, GWAS analysis has shown that dual 
specificity phosphatase 8 (DUSP8) and MOB2, respectively $\sim 140$ and $220 \mathrm{~kb}$ telomeric to KRTAP5-6, are expressed biallelically ${ }^{7}$. We note that our results correspond rather closely with the extent of a TAD detected by $\mathrm{Hi}-\mathrm{C}$ measurements (Supplementary Fig. 18) in EndoC- $\beta \mathrm{H} 1$ cells $^{15,38}$. Any analysis of gene regulation must consider the fact that the maternal and paternal chromosomes could have quite different interaction patterns over the entire domain.

This study began with an examination of the SNP rs2334499, strikingly associated with T2D susceptibility in an parent-oforigin-specific manner. Our results strongly suggest that this behavior is connected to significant differences in chromatin organization between maternal and paternal chromosomes extending from rs2334499 to the INS gene. The differences we observe in EndoC- $\beta \mathrm{H} 1$ cells are not directly coupled to rs 2334499 itself, as in these cells this SNP is homozygous. However, it seems likely that some SNP associated with the T allele of rs2334499 interacts with the extended specific domain structure that we describe, perhaps to affect INS expression in a parent-of-originspecific manner. The results presented here establish remarkable, complex and distinct higher structural organizations for the two alleles. The complex series of interactions connects the entire 460 $\mathrm{kb}$ region between KRTAP5-6 and INS, and strongly supports the idea that this is an extended locus with one set of interactions for the maternal and the other for the paternal allele. Although imprinting is observed centromeric to INS at sites such as KCNQ1, it seems likely that KRTAP5-6/INS constitutes a separately regulated domain.

Parent-of-origin-specific expression is typically associated directly or indirectly with parent-of-origin-specific DNA methylation ${ }^{39}$. Methylation of cytosine at certain $\mathrm{CpG}$ residues within a CTCF-binding site can disrupt its binding ${ }^{19}$. Because CTCF, through its recruitment of the cohesin complex, is well documented as helping to establish long-range contacts and 'loop' formation within the nucleus ${ }^{40}$, we looked for CTCF sites near KRTAP5-6 that might contribute to domain structure in the observed specific manner. A partially methylated region with seven CpGs, shown by Kong et al. ${ }^{7}$ to display a methylation pattern correlated with the risk variant of rs2334499, did not show allele-specific methylation patterns in EndoC- $\beta \mathrm{H} 1$ cells (Fig. 4a, orange-colored CpGs in Supplementary Fig. 6), which do not carry that variant. However, in agreement with their observations, we find that the fourth $\mathrm{CpG}$ is most highly methylated; in EndoC- $\beta \mathrm{H} 1$ cells methylation is nearly complete on both alleles (Fig. 4a). It should be noted that Kong et al. observed that the parental origin would not affect the association between rs2334499 and methylation levels of these CpGs.

Because those CTCF sites cannot be related to the observed parent-of-origin-specific chromatin organization of KRTAP5-6/ INS, we looked for other sites in the neighborhood and found a previously unrecognized CTCF-binding site (Supplementary Figs. 6 and 14). ChIP-PCR analysis (Fig. 4c) shows that this site is occupied by CTCF largely on the maternal allele, making it a plausible candidate for helping to organize KRTAP5-6/INS in a specific manner. This site is within a CpG island that is preferentially methylated on the paternal allele (Fig. $4 \mathrm{~b}$, red-colored CpGs in Supplementary Fig. 6), but the closest CpG is not at a location in the CTCF-binding motif that has been shown to disrupt CTCF-binding when methylated. Consistent with our methylation analysis, in a recently published dataset (http:// meltonlab.rc.fas.harvard.edu/data/pancreatic_enhancers/ $)^{41}$, these five $\mathrm{CpG}$ sites showed partial methylation pattern in mature $\beta$-cells (methylation level: 0.5, 0.286, 0.636, 0.538, 0.417), although the allelic preference was not reported (Supplementary Fig. $\left.19^{42}\right)$. The average methylation level $(0.475)$ is close to our results in EndoC- $\beta \mathrm{H} 1$ cells (0.444, Fig. $4 \mathrm{~b})$. Other cell types related to islet maturation process have higher methylation level. For example, in human pluripotent stem cells (hPSC), the methylation levels are $0.833,1,0.867,0.955,0.906$, and in pancreatic $\alpha$-cells, the levels are $1,0.667,0.9,0.444,0.636$. Our current study is limited to EndoC- $\beta \mathrm{H} 1$ cells due to limited resource and time. In future studies, it would be interesting to investigate whether the methylation pattern in normal primary pancreatic $\beta$ cells is consistent with that found in EndoC- $\beta \mathrm{H} 1$ cells, and whether the samples from diabetic patients would show a different pattern.

In our in vitro assay, we did not detect any effect of DMR CpG methylation on the affinity between CTCF and dsDNA (Supplementary Fig. 15). We suggest that the low level of CTCF binding to the paternal allele might reflect its high extended level of CpG methylation in this region (Fig. 4b), known to be associated with heterochromatin formation ${ }^{43}$ and therefore of reduced accessibility to transcription factors. This is supported by Lawlor et al. ${ }^{15}$ who assigned this CTCF site to the edge of a 37.2 $\mathrm{kb}$ "weak repressed Polycomb" heterochromatin region centromeric to OREG0020670. Compaction of chromatin, independent of CTCF binding, might contribute to parent-of-originspecific transcriptional behavior.

We observed that the histone 3 lysine 27 acetylation (H3K27ac) mark is enriched on the maternal allele (rs111402257 - C allele), the allele to which CTCF preferentially binds. On the other hand, we did not observe significant allelic bias of histone 3 lysine 9 trimethylation (H3K9me3) mark enrichment in this region (Supplementary Fig. 20). It is possible that another transcription factor binding site nearby would affect CTCF binding, and this transcription factor could be involved in DNA methylation. To aid future studies, we list the potential transcription factors that can bind to this region in Supplementary Table $2^{44}$.

We have identified a topologically discrete domain extending from KRTAP5-6 to INS, within which the two chromosomes have quite different interaction patterns, probably coupled to distinct DNA methylation patterns. One of these interactions, restricted to the paternal allele, directly connects the INS promoter to the KRTAP5-6 locus at the other end of the domain. Other interactions on the maternal allele connect KRTAP5-6 with H19 and the H19-ICR. This strongly supports the idea that the entire region is a single domain dominated by an imprinting mechanism. Parentof-origin-specific contacts between the H19 locus and IGF2 have been described in earlier studies in mouse cells ${ }^{45-47}$ and are connected with parent-of-origin-specific expression of H19 and IGF2. KRTAP5-6/INS is the larger domain which in humans encompasses all these contacts and differential expression patterns. Our results are summarized in Fig. 7.

The SNP rs2334499 is located at the telomeric end of KRTAP56/INS. The unusual property of the T allele, conferring opposite, parent-of-origin-specific T2D effects, was an important focus of the paper by Kong et al. ${ }^{7}$. The results we present here indicate that this SNP is located at the telomeric end of this large domain, and strongly suggests that within this domain there exist other SNPs that may confer allele and parent-of-origin-specific regulatory properties on expression of INS or other genes within the domain, such as $S Y T 8^{8}$. As in the case of rs2334499, the contributions of these SNPs to T2D susceptibility may not be detectible without considering the parental origin. Further GWAS studies with extended populations may reveal such SNPs.

Our observation that the three dimensional organization of the entire region between INS and KRTAP5-6 is parent-of-originspecific suggests that there would be parent-of-origin-specific effects on INS expression, an idea consistent with the parent-oforigin-specific effects on T2D susceptibility associated with SNP rs23344997. It has been accepted for many years that INS expression, unlike expression of the nearby IGF2 and H19 genes, 


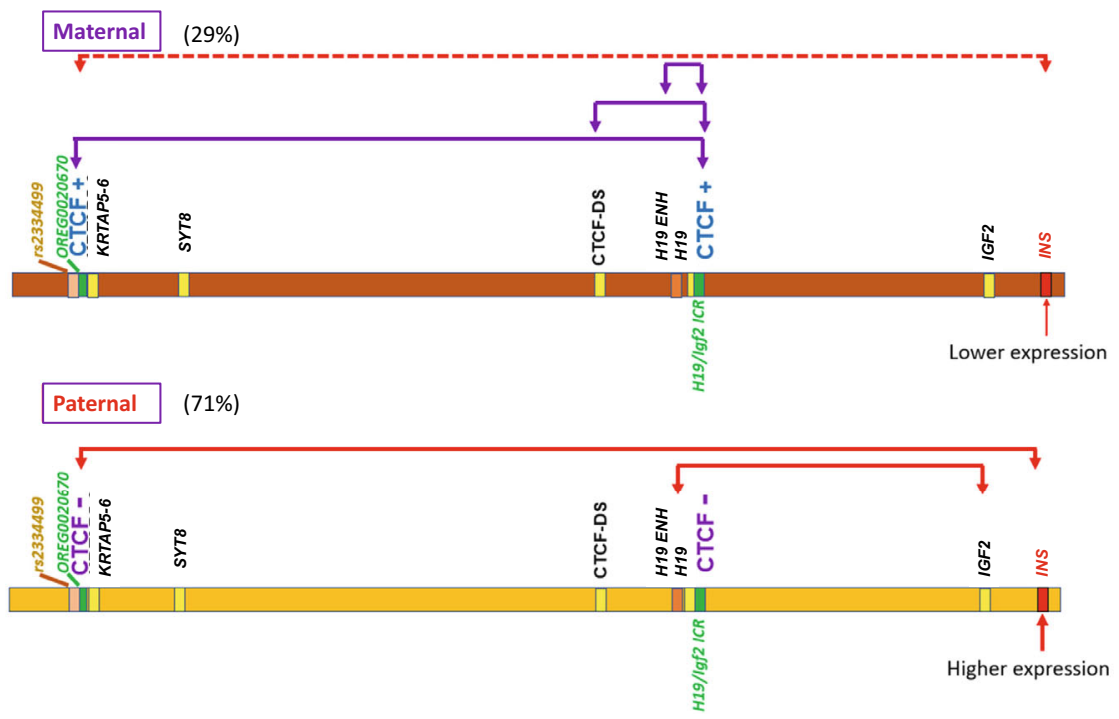

Fig. 7 Summary of the parent-of-origin-specific contact patterns in the region between INS and OREG0020670 on chromosome 11. The contacts are indicated above the chromosome. The binding preferences of CTCF sites are also indicated.

is not imprinted except in the yolk sac. However, it was proposed in 1996 that the parent-of-origin effect of T1D susceptibility at INS VNTR locus might be mediated by an imprinted domain outside INS VNTR ${ }^{30}$. Because the INS gene does not contain heterozygotic SNPs in EndoC- $\beta \mathrm{H} 1$ cells, we identified SNPs in read-through extended INS-IGF2 transcripts as a way of measuring allele specificity of expression of INS itself. These transcripts show a clear bias in favor of expression from the paternal INS gene. We were able to verify this result in a human sample, but in order to draw a general conclusion about the expression pattern of INS in the human population, many more samples must be tested. The INS promoter makes an unusual large number of genomic contacts (Supplementary Fig. 3a), and in this study we only analyzed parent-of-origin preference of one strong contact. While this result would indicate that the INS promoter in the two alleles are in different chemical environment, it would be prudent to not link parent-of-origin expression bias solely to the contact to KRTAP5-6 locus. Only after examining other strong contacts as well, we can get a better understanding of how gene imprinting mechanism can affect INS expression. In addition, the interpretation of these results depends on the assumption that the abundance of the INS-IGF2 read-through transcript is determined by the rate of transcription initiation at the INS promoter, and not by differences in rates of RNA splicing at a later stage, particularly important because the IGF2 gene itself is not expressed from the maternal allele ${ }^{48}$.

Our observations provide a tentative explanation of how sites at the telomere end of chromosome 11 near the gene KRTAP5-6 (Fig. 1a), which initially attracted our attention, can contain T2D diabetes susceptibility loci that display parent-of-origin-specific effects ${ }^{7}$ through interaction with large scale chromatin structure that differs dramatically on the two chromosomes. Thus, an imprinted locus can indirectly affect gene expression, recruiting distant regulatory elements in a parent-of-origin-specific manner. This may result in biased expression from one of the two chromosomes, as suggested above for the INS gene. An important conclusion of our study is that, because of the structural asymmetry involving contacts between INS and distant upstream elements, SNPs that affect INS expression should frequently have an overall effect that is parent-of-origin-specific. It seems likely that other imprinted loci will also help organize parent-of-originspecific chromatin contacts for a considerable distance around them, and that this will have significant regulatory implications throughout the genome. Hi-C or other studies that do not distinguish contributions from the individual alleles may overlook important differences in chromatin structure and regulation of gene expression.

\section{Methods}

Culture of EndoC- $\beta \mathrm{H} 1$ cells. EndoC- $\beta \mathrm{H} 1$ cell line was obtained via a material transfer agreement between Inserm Transfert, CNRS, Endocells, and National Institutes of Health. EndoC- $\beta \mathrm{H} 1$ cells were cultured in low-glucose Dulbecco's modified Eagle's medium (Life Technology, catalog no. 11885) with $2 \%$ bovine serum albumin (BSA, fatty acid free and heat shocked, Equitech, catalog no. BAH66), $50 \mu \mathrm{M}$ 2-mercaptoethanol, $10 \mathrm{mM}$ nicotinamide (Calbiochem, catalog no 481907), $5.5 \mu \mathrm{g} / \mathrm{ml}$ human transferrin (Sigma-Aldrich, catalog no. T8158), $6.7 \mathrm{ng} /$ $\mathrm{ml}$ sodium selenite (Sigma-Aldrich, catalog no. S9133), $100 \mathrm{U} / \mathrm{ml}$ penicillin, and $0.1 \mathrm{mg} / \mathrm{ml}$ streptomycin. Cells were seeded at a density of $6.2 \times 10^{5} / \mathrm{cm}^{2}$ on ECMgel (1\%) (Sigma-Aldrich, catalog no. E1270) /fibronectin $(2 \mu \mathrm{g} / \mathrm{ml}$; Sigma-Aldrich, catalog no. F1141) coated plates and cultured at $37^{\circ} \mathrm{C}$ in a $5 \% \mathrm{CO}_{2}$ incubator. The genomic coordination used in our experiments is based on Genome Reference Consortium Human Build 37 (GRCh37/hg19).

CRISPR/Cas 9 genome editing in EndoC- $\beta$ H1 cells. The procedure to perform genome editing of OREG0020670 in EndoC- $\beta \mathrm{H} 1$ cells was adapted from a recent protocoll ${ }^{17}$. A mixture of edited and un-edited cells is generated by this protocol, and currently there is no protocol to generate single clones in EndoC- $\beta \mathrm{H} 1$ cells. Specifically, 6 sgRNA sequences were designed from CRISPOR.org 49 and cloned into plentiCRISPRv2 vector ${ }^{50}$ (Addgene catalog no. 52961). The sgRNAs are designed to target three CTCF motifs (2 sgRNA sequences for each motif) in OREG0020670, at the 5'-end, middle and $3^{\prime}$-end of the element. The non-targeting control gRNA sequence is from Addgene Plasmid no. $80262^{51}$. The lentiviral vectors were produced in Lenti-X 293T cells (Takara catalog no. 632180). For 80\% confluency cells on one T175 flasks, a mixture of pMD2.G (6.85 $\mu \mathrm{g}$ ) (Addgene catalog no. 12259), psPAX2 (10.3 $\mu \mathrm{g}$ ) (Addgene catalog co. 12260), the respective cloned plentiCRISPRv2 $(12.85 \mu \mathrm{g})$ were for transfection with $100 \mu \mathrm{L}$ Lipofectamine LTX reagent (Invitrogen catalog no. 15338100). Growth medium was changed after $6 \mathrm{~h}$. The supernatant containing viral particles was collected $48 \mathrm{~h}$ after transfection. Fresh medium was added in and the supernatant was collected again after $24 \mathrm{~h}$. The viral particles were collected with Beckman Optima XL-100K ultracentrifuge at $29,000 \mathrm{rpm}$ for $2 \mathrm{~h}$ at $4^{\circ} \mathrm{C}$. The virus pellet was resuspended in $1.5 \% \mathrm{BSA}$ in phosphate buffered saline (PBS), and stored at $-80^{\circ} \mathrm{C}$ until use. EndoC- $\beta \mathrm{H} 1$ cells were transduced at a multiplicity of infection (MOI) of 8 , and the stable CRISPRedited EndoC- $\beta \mathrm{H} 1$ cells were generated after $4 \mathrm{ng} / \mu \mathrm{l}$ puromycin selection for 7 days. $8 \mathrm{ng} / \mu \mathrm{L}$ protamine was added into the medium during the lentiviral infection procedure. The CRISPR-editing efficiency was estimated by GeneArt Genomic Cleavage Detection Kit (Invitrogen catalog no. A24372).

$3 \mathrm{C}$ library preparation. The $3 \mathrm{C}$ library was prepared similar to our previous publications ${ }^{1,8}$. Specifically, $5 \times 10^{7}$ EndoC- $\beta$ H1 cells were trypsinized and resuspended in $10 \mathrm{~mL}$ growth medium, then fixed by $2 \%$ final concentration formaldehyde for $10 \mathrm{~min}$ at room temperature. The reaction was stopped by adding $2.5 \mathrm{M}$ glycine to a final concentration at $0.125 \mathrm{M}$ for $5 \mathrm{~min}$ at room temperature. 
The cells were collected by centrifugation and resuspended in lysis buffer $(10 \mathrm{mM}$ Tris- $\mathrm{HCl}, \mathrm{pH} 8.0,10 \mathrm{mM} \mathrm{NaCl}, 0.2 \% \mathrm{NP}-40$ ), and ruptured by a Dounce homogenizer on ice. The cross-linked nuclei was collected by centrifugation, and digested by BglII (New England Biolabs, catalog no. R0144) with the following procedure: resuspending $5 \times 10^{7}$ nuclei in $500 \mu \mathrm{L}$ NEBuffer 3.1 , and aliquoting $50 \mu \mathrm{L}$ nuclei to 10 new tubes; for each new tube adding $312 \mu \mathrm{L}$ NEBuffer 3.1 , and $38 \mu \mathrm{L} 1 \%$ sodium dodecyl sulfate (SDS) and incubating for $10 \mathrm{~min}$ at $65^{\circ} \mathrm{C}$; adding $44 \mu \mathrm{L} 10 \%$ Triton $\mathrm{X}-100$ and $400 \mathrm{U}$ BglII; incubating overnight at $37^{\circ} \mathrm{C}$. The digested chromatin was re-ligated by T4 DNA ligase (New England Biolabs, catalog no. M0202L) with the following procedure for each aliquot: adding $86 \mu \mathrm{L} 10 \%$ SDS and incubate for 30 min at $65^{\circ} \mathrm{C}$; transferring the liquid to a $50 \mathrm{~mL}$ conical tube; adding $745 \mu \mathrm{L} 10 \%$ Triton X-100, $745 \mu \mathrm{L} 10 \times$ ligation buffer $(500 \mathrm{mM}$ Tris-HCl, $\mathrm{pH} 7.5,100 \mathrm{mM}$ $\mathrm{MgCl}_{2}, 100 \mathrm{mM}$ dithiothreitol), $80 \mu \mathrm{L} 10 \mathrm{mg} / \mathrm{mL}$ BSA, $80 \mu \mathrm{L} 100 \mathrm{mM}$ ATP, $5960 \mu \mathrm{L}$ $\mathrm{dH}_{2} \mathrm{O}$; adding $7.5 \mu \mathrm{L}$ T4 DNA ligase and incubating overnight at $16^{\circ} \mathrm{C}$. The chromatin was de-crosslinked and digested with proteinase K (New England Biolabs, catalog no. P8107S) with the following procedure for each aliquot: adding $50 \mu \mathrm{l}$ $10 \mathrm{mg} / \mathrm{mL}$ proteinase $\mathrm{K}$ in $1 \times \mathrm{TE}$ buffer (10 mM Tris- $\mathrm{HCl}$, $\mathrm{pH} 8.0,1 \mathrm{mM}$ EDTA), and incubating overnight at $65^{\circ} \mathrm{C}$; adding an additional $50 \mu \mathrm{L} 10 \mathrm{mg} / \mathrm{mL}$ proteinase $\mathrm{K}$ in $1 \times \mathrm{TE}$ buffer, and incubate for $2 \mathrm{~h}$ at $42^{\circ} \mathrm{C}$. The $3 \mathrm{C}$ library was purified by phenol/chloroform extraction $(8 \mathrm{~mL}$ each) followed by ethanol precipitation $(0.1$ volume $3 \mathrm{M}$ sodium acetate, $\mathrm{pH} 5.3$ and 2.5 volume $100 \%$ ethanol, $2 \mathrm{~h}$ at $-80^{\circ} \mathrm{C}$ ). The pellet was redissolved in $400 \mu \mathrm{L} 1 \times \mathrm{TE}$ buffer, $\mathrm{pH} 8.0$, and was purified by phenol/chloroform extraction $(400 \mu \mathrm{L}$ each) followed by ethanol precipitation. The pellet was redissolved in $50 \mu \mathrm{L} 1 \times$ TE buffer. After finishing preparation of all the aliquots, the samples were pooled together, and $1 \mu 10 \mathrm{mg} / \mathrm{mL}$ RNase A (Thermo Scientific, catalog no. EN0531) was added following incubating the library for 15 min at $37^{\circ} \mathrm{C}$. The $3 \mathrm{C}$ library was stored at $-80^{\circ} \mathrm{C}$ before further experiments.

Genotyping of $\mathbf{3 C}$ interactions. The genotypes of $3 \mathrm{C}$ interactions were determined by PCR amplification with PicoMaxx High Fidelity PCR Master Mix (Agilent, catalog no. 600650). The 3C primers used in this study were listed in Supplementary Table 3. In order to improve the specificity of the PCR reactions, $1 \%$ dimethyl sulfoxide (DMSO) was added when necessary. The 3C PCR product was either sequenced directly after purification with QIAquick Gel Extraction Kit (Qiagen, catalog no. 28704), or cloned into pGEM-T vector (Promega, catalog no. A3600) by TA-cloning. For TA-cloning, the PCR product was ligated with pGEM-T vector and transformed into $E$. coli DH5a cells. We usually randomly picked $12-24$ colonies from the transformation agar plate and grew the $E$. coli culture in $10 \mathrm{~mL}$ LB Lenox medium overnight. The plasmids containing single cloned PCR product was extract with QIAprep Spin Miniprep Kit (Qiagen, catalog no. 27106), and was sent for Sanger sequencing to identify the alleles with BLAT ${ }^{52}$ alignment program (https:// genome-asia.ucsc.edu/cgi-bin/hgBlat?command=start). The sequences without insert, with no signal, with non-specific PCR amplification product and dual signal (double colonies) were excluded from analysis. We reported the number of alleles identified in the procedure instead of their percentage.

Chromatin immunoprecipitation (ChIP). ChIP experiments on CTCF were carried out using the ChIP-IT Express Kit (Active Motif, catalog no. 53008). Briefly, $1.5 \times 10^{7}$ cells were crosslinked by formaldehyde for $10 \mathrm{~min}$ at room temperature in the growth media, followed by $10 \mathrm{~min}$ incubation with glycine at room temperature to quench formaldehyde reactivity. Cells were washed twice with cold PBS, then the cell pellets were subjected to cell lysis by a Dounce homogenizer. The chromatin was sheared by a Bioruptor Plus sonication device to an average size of $\sim 500 \mathrm{bp}$. The chromatin immunoprecipitation procedures were carried out according to the manufacturer's instructions with an anti-CTCF antibody (Abcam, catalog no. ab70303), and $3 \mu \mathrm{L}$ antibody was used in each experiment (1:33 dilution). The crosslink was reversed by heating, and the protein was removed by proteinase $\mathrm{K}$ digestion. PCR reactions were performed with PicoMaxx High Fidelity PCR Master Mix (Agilent, catalog no. 600650) using primers specified in Supplementary Table 3. The genotype of the PCR product was examined by direct sequencing or colony sequencing after cloning into pGEM-T vector. ChIP experiments of histone H3K9me3 and H3K27ac were carried out similarly. The anti-histone H3K9me3 antibody (Abcam, catalog no. ab8898, 1:50 dilution) and anti-histone H3K27ac antibody (Abcam, catalog no. ab4729, 1:50 dilution) were used.

\begin{abstract}
Methylation analysis. The genomic DNA of EndoC- $\beta \mathrm{H} 1$ cells was prepared by AllPrep DNA/RNA Mini Kit (Qiagen, catalog no. 80204). The bisulfite conversion was carried out with EpiTect Fast Bisulfide Kit (Qiagen, catalog no. 59802) for DMR in OREG0020670 and EZ DNA Methylation-Lightning Kit (Zymo Research, catalog no. D5030S) for H19-ICR HS5. The freshly converted genomic DNA was used as the template for the PCR reactions using EpiTaq HS DNA polymerase (Takara, catalog no. R110B). The primers are listed in Supplementary Table 3. The amplification for DMR was achieved under the conditions that favoring elongation ( $3 \mathrm{mM} \mathrm{Mg}^{2+}, 0.2 \mathrm{mM}$ dNTP, $1 \mu \mathrm{M}$ primers and $2 \mathrm{~min}$ extension time). The PCR product was cloned into pGEM-T vector, and the individual clones were sequenced with a procedure similar to the 3C-PCR analysis above. The sequence analysis was performed with NCBI BLAST (https://blast.ncbi.nlm.nih.gov/Blast.cgi) ${ }^{53}$.
\end{abstract}

Genotyping INS-IGF2 isoform 1. Total RNA was extract from EndoC- $\beta \mathrm{H} 1$ cells by NucleoSpin RNA XS kit (Clontech, catalog no. 740902). The cDNA library was prepared from $5 \mu \mathrm{g}$ total RNA by Maxima H Minus Reverse Transcriptase (Thermo Scientific, catalog no. EP0751) following the recommended condition for long RT-PCR (> $5 \mathrm{~kb}$ ). The PCR product to genotype INS-IGF2 isoform 1 was amplified by KAPA HiFi HotStart polymerase (KAPA Biosystems, catalog no. KM2605). Due to the high GC content of the template, 5\% DMSO was added to the reaction. The product was verified after cloning into PGEM-T vector and sequenced by Sanger sequencing. The genotyping of the isoform was performed by direct sequencing the PCR product after agarose gel-purification.

CTCF gel-shift chemiluminescent EMSA assay (electrophoretic mobility shift assay). The CTCF gel-shift chemiluminescent EMSA assay was carried out with the Gel-shift Chemiluminescent EMSA Assay Kit (Active Motif, catalog no. 37341). The DNA oligo (purchased from Eurofins Genomics) sequence used was 5'gtagtgacccCGgagccaCGtggagccactttcagacccagagctgaagcaggagggtgacCGg-3'. The biotin-dT label was on the 5' end of the reverse strand. The CpGs that were either un-methylated or fully methylated are in underlined capitals. The CTCF-binding motif is in italic font. The EMSA assay was carried out following manufacture's instruction with some modifications. The final concentration for the labeled dsDNA was $2 \mathrm{nM}$. NP-40 was added to the final concentration of $0.02 \% .50 \mathrm{ng} / \mathrm{\mu L}$ Poly d(I-C) was used as a non-specific competitor. Maltose-binding protein-fused (MBP-fused) CTCF zinc finger 1-11 protein was over-expressed in E. coli and purified with amylose resin (New England Biolabs, catalog no. E8021S) according to the manufacturer's protocol. The details of the CTCF preparation procedure were described in a previous publication ${ }^{54}$.

Quantitative RT-PCR (qRT-PCR). To assess the effect of OREG0020670 on INS expression in EndoC- $\beta$ H1 cells, qRT-PCR experiments were performed on CRISPR-edited cells and control non-targeting gRNA treated cells, with Power SYBR $^{\mathrm{mx}}$ Green Cells-to-CT ${ }^{\mathrm{px}}$ Kit (Invitrogen catalog no. 4402954). GAPDH (glyceraldehyde-3-Phosphate dehydrogenase) endogenous control gene was used to normalize gene expression by the $\Delta \Delta \mathrm{Ct}$ method.

Human pancreatic islet. The human pancreatic islets sample was provided by the Integrated Islet Distribution Program (IIDP, catalog no. Pancreas-U.Miami4287_Islets, RRID:SAMN13866285) at City of Hope National Medical Center, which is funded by the National Institute of Diabetes and Digestive and Kidney Diseases (NIDDK), the National Institutes of Health (NIH Grant \#2UC4DK098085). The islet donor was a 30-years old non-diabetic female (https:// www.ncbi.nlm.nih.gov/biosample/SAMN13866285). The genomic DNA was extracted by Wizard Genomic DNA Purification Kit (Promega, catalog no. A1120). The IGF2 locus was genotyped by PCR amplification with PicoMaxx High Fidelity PCR Master Mix (Agilent, catalog no. 600650). The total RNA was extracted by RNeasy Mini Kit (Qiagen, catalog no. 74014). The allele-biased expression of IGF2 isoform 1 and INS-IGF2 isoform 1 was determined by RT-PCR, following the procedures mentioned above for EndoC- $\beta \mathrm{H} 1$ cells. All islets shipped via the IIDP are derived from cadaver donors. The IIDP documents informed consent for research purposes from donor relatives prior to offering the de-identified islets to established laboratories engaged in islet research and registered with IIDP. Research at NIH on de-identified specimens is not considered human subjects research (NHSR). Thus, no special permissions are needed.

Statistical analysis. Experimental data were analyzed using Graphpad QuickCalcs Web site (https://www.graphpad.com/quickcalcs/) and Microsoft Excel for Microsoft 365. Statistical comparison was conducted using the unpaired two-tailed Student's $t$ test. $P<0.05$ was considered statistically significant. Data were shown as mean \pm SD.

Reporting summary. Further information on experimental design is available in the Nature Research Reporting Summary linked to this paper.

\section{Data availability}

All data associated with this study are available from the corresponding authors upon reasonable request. Source data are provided with this paper. The $4 \mathrm{C}$-seq data used in this study ${ }^{1}$ are available in the National Center for Biotechnology Information Gene Expression Omnibus (GEO) database under accession code GSE112346. Source data are provided with this paper.

Received: 29 July 2020; Accepted: 28 June 2021; Published online: 15 July 2021

\section{References}

1. Jian, X. \& Felsenfeld, G. Insulin promoter in human pancreatic beta cells contacts diabetes susceptibility loci and regulates genes affecting insulin metabolism. Proc. Natl Acad. Sci. USA 115, E4633-E4641 (2018). 
2. Smith, A. C., Choufani, S., Ferreira, J. C. \& Weksberg, R. Growth regulation, imprinted genes, and chromosome 11p15.5. Pediatr. Res. 61, 43R-47R (2007)

3. DeChiara, T. M., Robertson, E. J. \& Efstratiadis, A. Parental imprinting of the mouse insulin-like growth factor II gene. Cell 64, 849-859 (1991).

4. Bartolomei, M. S., Zemel, S. \& Tilghman, S. M. Parental imprinting of the mouse H19 gene. Nature 351, 153-155 (1991).

5. Soejima, H. \& Higashimoto, K. Epigenetic and genetic alterations of the imprinting disorder Beckwith-Wiedemann syndrome and related disorders. J. Hum. Genet. 58, 402-409 (2013).

6. Moore, G. E. et al. Evidence that insulin is imprinted in the human yolk sac. Diabetes 50, 199-203 (2001).

7. Kong, A. et al. Parental origin of sequence variants associated with complex diseases. Nature 462, 868-874 (2009).

8. Xu, Z., Wei, G., Chepelev, I., Zhao, K. \& Felsenfeld, G. Mapping of INS promoter interactions reveals its role in long-range regulation of SYT8 transcription. Nat. Struct. Mol. Biol. 18, 372-378 (2011).

9. Xu, Z. et al. Mapping of long-range INS promoter interactions reveals a role for calcium-activated chloride channel ANO1 in insulin secretion. Proc. Natl Acad. Sci. USA 111, 16760-16765 (2014).

10. Ravassard, P. et al. A genetically engineered human pancreatic beta cell line exhibiting glucose-inducible insulin secretion. J. Clin. Invest. 121, 3589-3597 (2011).

11. Tsonkova, V. G. et al. The EndoC-betaH1 cell line is a valid model of human beta cells and applicable for screenings to identify novel drug target candidates. Mol. Metab. 8, 144-157 (2018).

12. Grarup, N., Sandholt, C. H., Hansen, T. \& Pedersen, O. Genetic susceptibility to type 2 diabetes and obesity: from genome-wide association studies to rare variants and beyond. Diabetologia 57, 1528-1541 (2014).

13. Lyssenko, V., Groop, L. \& Prasad, R. B. Genetics of type 2 diabetes: it matters from which parent we inherit the risk. Rev. Diabet. Stud. 12, 233-242 (2015).

14. Bonnefond, A. \& Froguel, P. Rare and common genetic events in type 2 diabetes: what should biologists know? Cell Metab. 21, 357-368 (2015).

15. Lawlor, N. et al. Multiomic profiling identifies cis-regulatory networks underlying human pancreatic beta cell identity and function. Cell Rep. $\mathbf{2 6}$ 788-801 e786. (2019).

16. Raviram, R. et al. 4C-ker: a method to reproducibly identify genome-wide interactions captured by $4 \mathrm{C}$-seq experiments. PLoS Comput. Biol. 12, e1004780 (2016).

17. Grotz, A. K. et al. A CRISPR/Cas9 genome editing pipeline in the EndoCbetaH1 cell line to study genes implicated in beta cell function. Wellcome Open Res. 4, 150 (2019).

18. Nativio, R. et al. Cohesin is required for higher-order chromatin conformation at the imprinted IGF2-H19 locus. PLoS Genet. 5, el000739 (2009).

19. Bell, A. C. \& Felsenfeld, G. Methylation of a CTCF-dependent boundary controls imprinted expression of the Igf2 gene. Nature 405, 482-485 (2000).

20. Hark, A. T. et al. CTCF mediates methylation-sensitive enhancer-blocking activity at the H19/Igf2 locus. Nature 405, 486-489 (2000).

21. Renda, M. et al. Critical DNA binding interactions of the insulator protein CTCF: a small number of zinc fingers mediate strong binding, and a single finger-DNA interaction controls binding at imprinted loci. J. Biol. Chem. 282, 33336-33345 (2007).

22. Ziebarth, J. D., Bhattacharya, A. \& Cui, Y. CTCFBSDB 2.0: a database for CTCF-binding sites and genome organization. Nucleic Acids Res. 41, D188-D194 (2013).

23. Ghirlando, R. \& Felsenfeld, G. CTCF: making the right connections. Genes Dev. 30, 881-891 (2016)

24. Monk, D. et al. Imprinting of IGF2 P0 transcript and novel alternatively spliced INS-IGF2 isoforms show differences between mouse and human. Hum. Mol. Genet 15, 1259-1269 (2006).

25. Johannessen, L. E. et al. Upregulation of INS-IGF2 read-through expression and identification of a novel INS-IGF2 splice variant in insulinomas. Oncol. Rep. 36, 2653-2662 (2016).

26. Kanatsuna, N. et al. Autoimmunity against INS-IGF2 protein expressed in human pancreatic islets. J. Biol. Chem. 288, 29013-29023 (2013).

27. Kanatsuna, N. et al. Doubly reactive INS-IGF2 autoantibodies in children with newly diagnosed autoimmune (type 1) diabetes. Scand. J. Immunol. 82, 361-369 (2015).

28. Wernersson, R. et al. Analysis artefacts of the INS-IGF2 fusion transcript BMC Mol. Biol. 16, 13 (2015).

29. Wasserfall, C. et al. Persistence of pancreatic Insulin mRNA expression and proinsulin protein in type 1 diabetes pancreata. Cell Metab. 26, 568-575 e563. (2017).

30. Bennett, S. T. \& Todd, J. A. Human type 1 diabetes and the insulin gene: principles of mapping polygenes. Annu Rev. Genet. 30, 343-370 (1996).

31. Kennedy, G. C., German, M. S. \& Rutter, W. J. The minisatellite in the diabetes susceptibility locus IDDM2 regulates insulin transcription. Nat. Genet. 9, 293-298 (1995)
32. Mutskov, V. \& Felsenfeld, G. The human insulin gene is part of a large open chromatin domain specific for human islets. Proc. Natl Acad. Sci. USA 106, 17419-17424 (2009).

33. Xiao, T., Li, X. \& Felsenfeld, G. The Myc-associated zinc finger protein (MAZ) works together with CTCF to control cohesin positioning and genome organization. Proc. Natl. Acad. Sci. USA. 118, e2023127118 (2021).

34. Bennett, S. T. et al. Susceptibility to human type 1 diabetes at IDDM2 is determined by tandem repeat variation at the insulin gene minisatellite locus. Nat. Genet. 9, 284-292 (1995).

35. Gong, H. et al. An updated nomenclature for keratin-associated proteins (KAPs). Int J. Biol. Sci. 8, 258-264 (2012).

36. Rogers, M. A., Langbein, L., Praetzel-Wunder, S., Winter, H. \& Schweizer, J. Human hair keratin-associated proteins (KAPs). Int Rev. Cytol. 251, 209-263 (2006).

37. Uhlen, M. et al. Proteomics. Tissue-based map of the human proteome. Science 347, 1260419 (2015)

38. Robinson, J. T. et al. Juicebox.js provides a cloud-based visualization system for Hi-C data. Cell Syst. 6, 256-258 e251. (2018).

39. Adalsteinsson, B. T. \& Ferguson-Smith, A. C. Epigenetic control of the genome-lessons from genomic imprinting. Genes 5, 635-655 (2014).

40. Merkenschlager, M. \& Nora, E. P. CTCF and cohesin in genome folding and transcriptional gene regulation. Annu. Rev. Genomics Hum. Genet. 17, 17-43 (2016).

41. Alvarez-Dominguez, J. R. et al. Circadian entrainment triggers maturation of human in vitro islets. Cell Stem Cell 26, 108-122 e110. (2020).

42. Kent, W. J. et al. The human genome browser at UCSC. Genome Res. 12, 996-1006 (2002).

43. Cedar, H. \& Bergman, Y. Linking DNA methylation and histone modification: patterns and paradigms. Nat. Rev. Genet. 10, 295-304 (2009).

44. Cartharius, K. et al. MatInspector and beyond: promoter analysis based on transcription factor binding sites. Bioinformatics 21, 2933-2942 (2005).

45. Murrell, A., Heeson, S. \& Reik, W. Interaction between differentially methylated regions partitions the imprinted genes Igf2 and $\mathrm{H} 19$ into parentspecific chromatin loops. Nat. Genet. 36, 889-893 (2004).

46. Kurukuti, S. et al. CTCF binding at the $\mathrm{H} 19$ imprinting control region mediates maternally inherited higher-order chromatin conformation to restrict enhancer access to Igf2. Proc. Natl Acad. Sci. USA 103, 10684-10689 (2006).

47. Yoon, Y. S. et al. Analysis of the H19ICR insulator. Mol. Cell Biol. 27 , 3499-3510 (2007)

48. Bergman, D., Halje, M., Nordin, M. \& Engstrom, W. Insulin-like growth factor 2 in development and disease: a mini-review. Gerontology 59, 240-249 (2013).

49. Concordet, J. P. \& Haeussler, M. CRISPOR: intuitive guide selection for CRISPR/Cas9 genome editing experiments and screens. Nucleic Acids Res. 46 W242-W245 (2018).

50. Sanjana, N. E., Shalem, O. \& Zhang, F. Improved vectors and genome-wide libraries for CRISPR screening. Nat. Methods 11, 783-784 (2014).

51. Doench, J. G. et al. Optimized sgRNA design to maximize activity and minimize off-target effects of CRISPR-Cas9. Nat. Biotechnol. 34, 184-191 (2016).

52. Kent, W. J. BLAT-the BLAST-like alignment tool. Genome Res. 12, 656-664 (2002).

53. Altschul, S. F., Gish, W., Miller, W., Myers, E. W. \& Lipman, D. J. Basic local alignment search tool. J. Mol. Biol. 215, 403-410 (1990).

54. Xiao, T., Wongtrakoongate, P., Trainor, C. \& Felsenfeld, G. CTCF recruits centromeric protein CENP-E to the pericentromeric/centromeric regions of chromosomes through unusual CTCF-binding sites. Cell Rep. 12, 1704-1714 (2015).

\section{Acknowledgements}

This work was supported by the Intramural Research Program of the National Institute of Diabetes and Digestive and Kidney Diseases, National Institutes of Health. We thank Inserm Transfert, CNRS and Endocells for providing the pancreatic $\beta$-cell line, EndoC$\beta$ H1. We thank Dr. Yihong Ye and Dr. Xin Li for their help in the revision process.

\section{Author contributions}

X.J. and G.F. designed research; X.J. performed research; X.J. and G.F. analyzed data; and X.J. and G.F. wrote the paper.

\section{Funding}

Open Access funding provided by the National Institutes of Health (NIH).

\section{Competing interests}

The authors declare no competing interests. 


\section{Additional information}

Supplementary information The online version contains supplementary material available at https://doi.org/10.1038/s41467-021-24635-2.

Correspondence and requests for materials should be addressed to G.F.

Peer review information Nature Communications thanks Anita Göndör, Adele Murrell, and other, anonymous reviewers for their contributions to the peer review of this work. Peer review reports are available.

Reprints and permission information is available at http://www.nature.com/reprints

Publisher's note Springer Nature remains neutral with regard to jurisdictional claims in published maps and institutional affiliations. (c) (P) Open Access This article is licensed under a Creative Commons Attribution 4.0 International License, which permits use, sharing, adaptation, distribution and reproduction in any medium or format, as long as you give appropriate credit to the original author(s) and the source, provide a link to the Creative Commons license, and indicate if changes were made. The images or other third party material in this article are included in the article's Creative Commons license, unless indicated otherwise in a credit line to the material. If material is not included in the article's Creative Commons license and your intended use is not permitted by statutory regulation or exceeds the permitted use, you will need to obtain permission directly from the copyright holder. To view a copy of this license, visit http://creativecommons.org/ licenses/by/4.0/.

This is a U.S. government work and not under copyright protection in the U.S.; foreign copyright protection may apply 2021 